\title{
Topology Control with Application Constraints
}

\author{
Michael Stein*, Géza Kulcsár ${ }^{\dagger}$, Immanuel Schweizer*, Gergely Varró ${ }^{\dagger}$, Andy Schürr ${ }^{\dagger}$ and Max Mühlhäuser* \\ Technische Universität Darmstadt, Germany \\ *\{michael.stein, schweizer, max $\} @$ tk.informatik.tu-darmstadt.de \\ $\dagger\{$ geza.kulcsar, gergely.varro, andy.schuerr $\} @$ es.tu-darmstadt.de
}

\begin{abstract}
Numerous topology control algorithms for wireless sensor networks exist. Typically, these algorithms optimize general network metrics like the transmission range of sensors. Being of general nature, they suit application-specific requirements insufficiently; e.g., application-specific communication patterns may favor links that the general algorithm will remove.

We suggest a new research focus called application-specific topology control and propose a methodic approach for which we provide a first exploration. First, application-specific communication patterns are expressed as overlay graphs. Then, applicationspecific requirements are specified as constraints on this overlay. Next follows the core of the method, geared toward deriving application-specific topology control algorithms.

Applying the method to a data collection application with a many-to-one communication pattern, we propose two topology control algorithms. In a simulation study, we evaluate these algorithms against the existing algorithm kTC and a shortest path tree, showing that the method allows trading off general network optimization against application-specific optimization.
\end{abstract}

Index Terms-topology control, WSN, application-specific

\section{INTRODUCTION}

Many topology control algorithms have been presented during the last decade [1]. Today, topology control algorithms are designed for all-to-all communication, thus, optimizing general network metrics like the transmission range of the nodes. However, wireless sensor networks (WSNs) are application oriented [2]. Most applications in WSNs follow specific communication patterns, e.g., the many-to-one pattern with all nodes sending data to a common base station. The corresponding application-specific communication overlays are not considered for topology control decisions in even the most practical algorithms. Hence, application-specific communication links might be removed, leading to severe implications for application performance.

Application-specific topology control has been identified as future work already in 2008 by Wang [1]. Xing et al. [3] propose the CTC algorithm, emphasizing that the required level of topology quality in a WSN depends on the application. CTC can be configured accordingly. However, CTC is unaware of the specific communication patterns introduced by different applications. The paper at hand is the first to explore application-specific topology control in depth. We model the application as an overlay graph to describe application-specific communication patterns. Application requirements can then be described as constraints on the overlay. Moreover, we propose a generic method to derive application-specific topology control algorithms from conventional topology control algorithms.
As a case study, we focus on the prevalent data collection application, which relies on the many-to-one communication pattern. Applying the overlay model and the generic method, we propose two application-specific topology control algorithms based on the existing kTC [4] algorithm.

We conduct a simulative evaluation of these algorithms against kTC and a shortest path tree as baseline. The evaluation illustrates that our application-specific algorithms preserve application-specific links and are still able to optimize the network for general communication. Demonstrating the tradeoff between application-specific optimization and general optimization, we show that our new algorithms can be configured to control this trade-off as desired.

\section{SySTEM MODEL}

In wireless networks, all nodes typically send with maximum transmission power, reaching as many nodes as possible. Employing topology control, each node reduces the number of neighbors such that the resulting topology is still connected and has beneficial properties. This edge reduction allows nodes to reduce their transmission power, hence, saving energy.

Topology control is usually employed on the underlay only. The underlay is an undirected graph $G_{U}=\left(V, E_{U}\right)$ in which two nodes $u, v \in V$ are connected by an edge $(u, v) \in E_{U}$ if there is a physical link between them. An example of an underlay is depicted in Fig. 1 by black solid lines.

A WSN is usually deployed to serve one application [2]. An application induces an overlay $G_{O}=\left(V, E_{O}\right)$, which reflects the communication pattern of the application. An example of an overlay is depicted in Fig. 1 by dashed lines.

The contribution of this paper builds upon the observation that topology control increases distances on the overlay, thus, affecting application performance. This can be observed in Fig. 1, where four nodes $\left(v_{1}, v_{2}, v_{3}, v_{4}\right)$ send data to a base station (b). After execution of the kTC algorithm, which removes two edges $e_{1}$ and $e_{2}$ from the graph, the hop count to $b$ increases for all nodes but for $v_{3}$.

In order to provide good application performance, topology control must ensure that the distance increase in the overlay is sufficiently small. Based on the underlay $G_{U}$, each overlay edge $(u, v) \in E_{O}$ has a distance weight denoted by $\mathcal{D}_{\mathcal{M}}\left(G_{U}, u, v\right)$, where $\mathcal{M}: E_{U} \rightarrow \mathcal{R}$ is an overlay distance metric, which assigns an application-specific routing weight to underlay edges. $\mathcal{D}_{\mathcal{M}}\left(G_{U}, u, v\right)$ is the minimum distance over all paths between $u$ and $v$ in $G_{U}$. For a path $v_{1} \ldots v_{n}$, we define the distance as the sum of the underlay edge weights. 


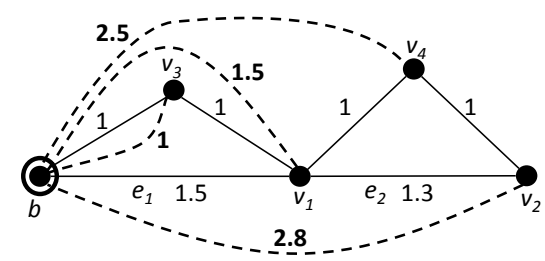

Fig. 1. Example topology with Euclidean edge weights.

Typically, the overlay distance metric $\mathcal{M}$ is defined based on the application requirements. For example, applications relying on strict delay constraints, like factory automation systems [5], mainly use the number of hops between nodes to specify the costs of routing paths. Other applications aim at high energy efficiency. These applications specify the cost of each link based on the associated energy consumption. For the sake of simplicity, we use the Euclidean distance between two nodes to specify the cost of the edge $e \in E_{U}$, i.e., $\mathcal{M}(e)=\|e\|$.

\section{APPLICATION-SPECIFIC TOPOLOGY CONTROL}

This section presents our approach to application-specific topology control. First, we provide a generic method. This method is then applied to the many-to-one communication pattern, which is found in most WSN applications.

We aim at achieving both sufficient general network performance and good application performance. Therefore, we suggest to execute a conventional topology control algorithm, but define application constraints to limit the edge removal to edges that are not specific to the application. The corresponding method consists of three sequentially conducted phases:

Phase I: Edge marking. A conventional topology control algorithm is executed. However, instead of removing edges immediately, the edges are marked for further processing.

Phase II: Removal candidate edge selection. Based on the set of marked edges, an edge selection strategy selects a subset of removal candidate edges. This strategy considers graph constraints, which reflect application-specific requirements.

Phase III: Edge removal. Only the removal candidate edges are then removed from $E_{U}$.

In the following, $E^{m} \subseteq E_{U}$ and $E^{r} \subseteq E^{m}$ denote the sets of marked edges and removal candidate edges, respectively.

This is a generic method, hence, there is some degree of freedom. First, a topology control algorithm has to be selected for the execution in Phase I. Second, an edge selection strategy needs to be specified. For the second aspect, we suggest to model both the network and the application as graphs as done in Section II. This allows for defining application-specific constraints in an efficient way. These constraints can then be used to develop the edge selection strategy.

For the rest of this paper, we will focus on the many-toone communication pattern. This communication pattern is often used in WSN applications because sensor nodes typically report collected data to a central base station. We will first model the data collection application as an overlay and define a corresponding application constraint. Afterwards, we will select an algorithm for the execution in Phase I, and provide two edge selection strategies to ensure this constraint.

We model the data collection application as follows.

Definition 1. For the data collection application, the overlay is an undirected graph $G_{O}=\left(V, E_{O}\right)$ in which all nodes $v_{i} \in V \backslash\{b\}$ are connected to the base station by an overlay edge $e_{i} \in E_{O}$.

An example of such an overlay is again depicted in Fig. 1.

As noted before, we preserve application-specific edges by introducing constraints on the overlay. For the many-to-one communication pattern, we specify the following constraint.

Constraint 1. $G_{U}^{o l d}=\left(V, E_{U}\right)$ and $G_{U}^{\text {new }}=\left(V, E_{U} \backslash E^{r}\right)$ are the underlay graphs before and after the execution of topology control, respectively. For a base station $b \in V$, an overlay distance metric $\mathcal{M}$ and a stretch parameter $a \in[1, \infty[$,

$$
\forall v \in V: \frac{\mathcal{D}_{\mathcal{M}}\left(G_{U}^{\text {new }}, v, b\right)}{\mathcal{D}_{\mathcal{M}}\left(G_{U}^{\text {old }}, v, b\right)} \leq a .
$$

A low overlay distance is assumed to indicate good application performance. Constraint 1 specifies that the overlay distance from each node to the base station must not increase by more than a given stretch parameter $a$.

In order to mark edges in Phase I, we use kTC [4], a state of the art topology control algorithm. $\mathrm{kTC}$ removes the longest edge of each triangle in $G_{U}$ if it is at least $k$ times longer than the shortest edge in the triangle. We denote the resulting underlay by $G_{\mathrm{kTC}}$. It is important to note that any other topology control algorithm can be used to mark edges.

The challenging part of our proposed method is to provide a suitable edge selection strategy. The main difficulty of edge selection is the huge solution space: As the edge selection strategy may output any subset $E^{r} \subseteq E^{m}$, there are in total $2^{\left|E^{m}\right|}$ possible selections. Moreover, the removal decisions for different edges are inter-dependent because a path on the overlay typically employs multiple edges on the underlay. In other words, the decision if a certain edge can be removed without violating the overlay constraints typically depends on whether other marked edges are removed as well.

There are two intuitive assumptions that can be made when making the removal decision for an edge $e \in E^{m}$. We can either assume that the other marked edges $E^{m} \backslash e$ are preserved, or we can assume that these edges are removed. These assumptions reflect the core ideas of our two edge selection algorithms, which are presented in the following.

A. $1-\mathrm{kTC}$

The first edge selection strategy, local-kTC ( $1-\mathrm{kTC}$ ), builds upon the assumption that none of the marked edges but the investigated local one will be removed in Phase III. First, the algorithm computes the overlay distance $\mathcal{D}_{\mathcal{M}}\left(G_{U}, v, b\right)$ from each node $v \in V$ to the base station $b$. For each marked edge, $1-\mathrm{kTC}$ temporarily removes the edge from the underlay. Then, it updates the distances of the incident nodes to $b$ and readds the edge to the underlay. If the increase in distance for the incident nodes is less than or equal to the stretch parameter $a$, 


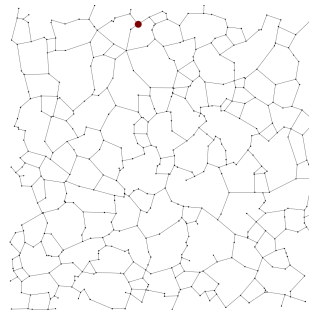

(a) $\mathrm{kTC}$

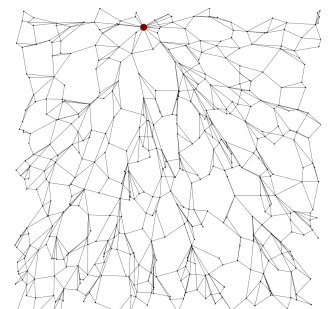

(b) $1-\mathrm{kTC}(a=1.0)$
Fig. 2. kTC and $1-k T C$ topologies. The base station $b$ is marked red.

the edge becomes a removal candidate. The resulting underlay after Phase III is denoted by $G_{1-\mathrm{kTC}}$.

For example, executing $1-\mathrm{kTC}(a=1.4)$ for the topology shown in Fig. 1 would confirm the removal of both $e_{1}$ and $e_{2}$.

\section{B. $\mathrm{g}-\mathrm{kTC}$}

The second edge selection strategy, global-kTC $(g-k T C)$, differs from $1-k T C$ regarding the assumption being made about the removal decisions of other marked edges. When $g-k T C$ investigates the implications of the removal of an edge $e \in E^{m}$, it expects that globally all other marked edges will be removed as well. First, just like $1-\mathrm{kTC}$, $g-k$ TC computes the overlay distance from each node to the base station. Then, the algorithm temporarily removes all marked edges from the underlay. Next, $g-k T C$ iterates over all marked edges, recomputes the overlay distances for the incident nodes and adds an edge to the set of removal candidates only if the increase in overlay distance is less than the stretch parameter $a$ for both incident nodes. Finally, the algorithm re-adds all marked edges to the underlay. The resulting underlay after Phase III is denoted by $G_{\mathrm{g}-\mathrm{kTC}}$.

For example, executing $\mathrm{g}-\mathrm{kTC}(a=1.4)$ for the topology shown in Fig. 1 would remove $e_{2}$ and preserve $e_{1}$.

\section{Theoretical ANALYsis}

This section provides a theoretical analysis of the algorithms introduced in Section III. A key result is that $G_{\mathrm{kTC}}$ is a subgraph of both $G_{1-\mathrm{kTC}}$ and $G_{\mathrm{g}-\mathrm{kTC}}$. This subgraph relation has two important implications. First, as $G_{\mathrm{kTC}}$ is connected [4], $G_{1-\mathrm{kTC}}$ and $G_{\mathrm{g}-\mathrm{kTC}}$ are connected. Second, each path that exists in $G_{\mathrm{kTC}}$ does also exist in $G_{1-\mathrm{kTC}}$ and $G_{\mathrm{g}-\mathrm{kTC}}$. Thus, the distances between arbitrary nodes in $G_{1-\mathrm{kTC}}$ or $G_{\mathrm{g}-\mathrm{kTC}}$ are bounded by those in $G_{\mathrm{kTC}}$. Additionally, we can prove for $g-k$ TC by induction ${ }^{1}$ that Constraint 1 is fulfilled.

While $1-\mathrm{kTC}$ and $\mathrm{g}-\mathrm{kTC}$ retain connectivity and symmetry from kTC, it is also important to state that $G_{1-\mathrm{kTC}}$ and $G_{\mathrm{g}-\mathrm{kTC}}$ are neither degree-bounded nor planar, while $G_{\mathrm{kTC}}$ is [4]. Moreover, in contrast to kTC [4], $G_{1-\mathrm{kTC}}$ and $G_{\mathrm{g}-\mathrm{kTC}}$ are not $\theta$-separated, i.e., it cannot be shown that two logical neighbors are separated by at least an angle of $\theta$. This means, $1-\mathrm{kTC}$ and $\mathrm{g}-\mathrm{kTC}$ lose some of the theoretical properties provided by kTC. However, we argue that the application specifies the

\footnotetext{
${ }^{1}$ The proof has been omitted due to space restrictions.
}

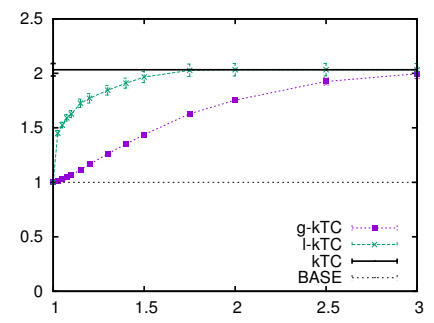

(a) Base stretch

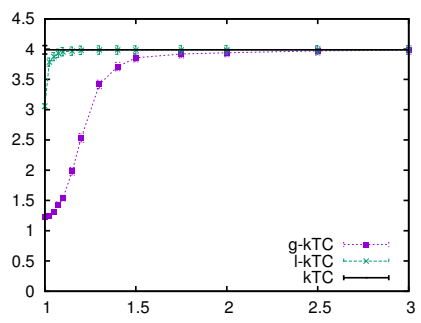

(b) General stretch
Fig. 3. Length stretch factors (x-axis: stretch parameter $a$ )

key requirements for topology control. If a theoretical property is important for the application, this should be encoded into the topology control constraints provided by the application.

\section{EVALUATion}

This section evaluates our approach to application-specific topology control. We state three main hypotheses:

1) $1-k T C$ and $g-k T C$ promise better application performance than KTC in terms of overlay distance between the sensor nodes and the base station.

2) Building on the general algorithm $\mathrm{kTC}, 1-\mathrm{kTC}$ and $g-k$ TC still achieve good general network performance.

3) There exists a trade-off between general performance and application-specific performance, which can be controlled with the stretch parameter $a$.

In order to evaluate these hypotheses, we conducted a simulation study, which is presented in the following.

\section{A. Simulation Setup}

We used an integrated simulation environment based on the network simulator PeerfactSim.KOM [6] to generate topologies and the graph transformation tool eMoflon [7] to execute topology control ${ }^{2}$. We simulated 500 nodes including one base station. The nodes are placed uniformly at random onto a squared plane with a side length of 10 units, and each node has a maximum transmission range of 1 unit. Each simulation setup was executed 200 times with different random seeds. All results are given with $95 \%$ confidence intervals.

We compare $1-\mathrm{kTC}$ and $\mathrm{g}-\mathrm{kTC}$ to $\mathrm{kTC}$ and the UDG. $\mathrm{kTC}$ was always executed with $k=1.0$. Additionally, we consider the most intuitive application-specific topology, called BASE, which is a shortest path tree rooted at the base station.

\section{B. Hypothesis 1: Application Performance}

In our case study, we focus on overlays with the manyto-one communication pattern (cf. Definition 1). Thus, the stretch factor for the overlay distance from the sensor nodes to the base station is the most important metric to measure the application performance. In the following, we denote the stretch factor that reflects the maximum increase in distance over all $|V|-1$ sensor nodes to the base station by the term

\footnotetext{
${ }^{2}$ For more details about graph transformation and the corresponding model, please refer to [8].
} 
base stretch factor. The base stretch factors for the different topologies are given in Fig. 3a.

BASE reflects the application-specific optimum. Creating a shortest path tree rooted at the base station, BASE has a base stretch factor of 1 . On the other hand, kTC is applicationagnostic, resulting in the removal of many edges (cf. Fig. 2a) and a base stretch factor of slightly above 2 . It must be expected that this increase in path length degrades the application performance significantly compared to the UDG.

$1-k T C$ and $g-k T C$ build upon $\mathrm{kTC}$ but also consider Constraint 1. Consequently, both algorithms promise better application performance than $\mathrm{kTC}$. For a small stretch parameter $a, 1-\mathrm{kTC}$ and $\mathrm{g}-\mathrm{kTC}$ achieve a stretch factor close to 1. Increasing $a$ relaxes the overlay constraint, which leads to the removal of more application-specific edges and, thus, to an increase of the base stretch factor. Nevertheless, as $G_{\mathrm{kTC}}$ is a subgraph of $G_{1-\mathrm{kTC}}$ and $G_{\mathrm{g}-\mathrm{kTC}}$, their stretch factors are bounded by the ones of kTC. Moreover, $g-k T C$ even guarantees that the base stretch factor is bounded by $a$.

\section{Hypothesis 2: General Performance}

For general network performance, it is crucial to provide short paths for all-to-all communication. We measure the general stretch factor, which gives the maximum increase in path length between all $|V|(|V|-1) / 2$ pairs of nodes. Fig. 3b gives the general stretch factors of $k T C, 1-k T C$ and $g-k T C$.

As kTC strives for general network optimization, it also provides a reasonable general stretch factor. BASE creates a tree topology that is solely optimized for the data collection application. Having a general stretch factor of 205.16, the resulting topology is inappropriate for all-to-all communication.

Additionally, we notice that the general stretch factors of $1-k T C$ and $g-k T C$ are slightly smaller than the ones of kTC. The reason for this is that the preserved application-specific edges may also be used for all-to-all communication.

A wireless node may shrink its transmission power such that the node still reaches its farthest neighbor. The average maximum transmission range gives the average Euclidean distance from nodes to their farthest neighbor. A small value is beneficial because the required transmission power increases with distance. Also, small transmission power reduces network contention. For these reasons, we consider transmission range reduction to be the main target of topology control.

All topology control algorithms achieve significant transmission range reductions (Fig. 4). For the initial UDG, the average maximum transmission range is slightly above 0.96 . $\mathrm{kTC}$ reduces this value to about 0.46 . This reflects a reduction of more than $50 \%$ compared to the UDG. BASE achieves a transmission range reduction of around $25 \%$.

Recall that $G_{\mathrm{kTC}}$ is a subgraph of $G_{1-\mathrm{kTC}}$ and $G_{\mathrm{g}-\mathrm{kTC}}$. Therefore, $g-k T C$ and $1-k T C$ cannot outperform kTC in terms of transmission range reduction. Fig. $2 b$ demonstrates that $1-k$ TC preserves only those edges that point toward the base station, creating an intuitive visual pattern. Consequently, even for a small stretch parameter $(a \leq 1.1)$, the transmission range reduction is very close to the one of kTC. This is unfortunately

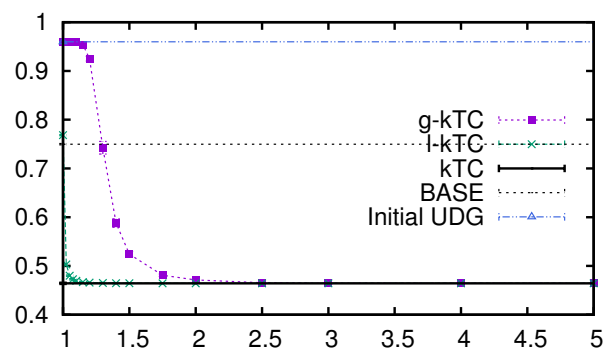

Fig. 4. Average maximum transmission range (x-axis: stretch parameter $a$ )

not the case for $\mathrm{g}-\mathrm{kTC}$, which requires strong relaxation of Constraint 1 for high transmission range reduction.

\section{Hypothesis 3: Trade-off}

kTC and BASE are the extreme cases that focus solely on general network performance and application performance, respectively. Consequently, neither kTC nor BASE perform well for both. It becomes evident that application-specific performance and general performance are competing optimization goals: They constitute a trade-off. $1-\mathrm{kTC}$ and $\mathrm{g}-\mathrm{kTC}$ aim at balancing this trade-off. As both algorithms build upon a conventional algorithm but consider application constraints, l-kTC and g-kTC are able to optimize the application without making all-to-all communication infeasible.

For $1-\mathrm{kTC}$ and $\mathrm{g}-\mathrm{kTC}$, the stretch parameter $a$ adjusts the aggressiveness. Low aggressiveness leads to an applicationspecific topology, whereas high aggressiveness improves general network performance. Consequently, we are able to control the trade-off above as desired.

In summary, we explored application-specific topology control. As the evaluation showed, this is possible with a trade-off between application-specific and general performance.

\section{ACKNOWLEDGMENT}

This work has been funded by the German Research Foundation (DFG) as part of project A01 within the Collaborative Research Center (CRC) 1053 - MAKI.

\section{REFERENCES}

[1] Y. Wang, "Topology Control for Wireless Sensor Networks," in Wireless Sensor Networks and Applications, 2008, pp. 113-147.

[2] J. Yick, B. Mukherjee, and D. Ghosal, "Wireless sensor network survey," Computer Networks, vol. 52, no. 12, pp. 2292-2330, 2008.

[3] G. Xing, C. Lu, X. Jia, and R. Pless, "Localized and configurable topology control in lossy wireless sensor networks," Ad Hoc Networks, vol. 11, no. 4, pp. 1345-1358, 2013.

[4] I. Schweizer, M. Wagner, D. Bradler, M. Mühlhäuser, and T. Strufe, "kTC - Robust and Adaptive Wireless Ad-hoc Topology Control," in ICCCN, 2012, pp. 1-9.

[5] A. Kumar Somappa, K. Øvsthus, and L. Kristensen, "An Industrial Perspective on Wireless Sensor Networks - A Survey of Requirements, Protocols, and Challenges," Communications Surveys Tutorials, vol. 16, no. 3, pp. 1391-1412, 2014.

[6] D. Stingl, C. Gross, J. Ruckert, L. Nobach, A. Kovacevic, and R. Steinmetz, "PeerfactSim.KOM: A Simulation Framework for Peer-to-Peer Systems," in HPCS, 2011, pp. 577-584.

[7] eMoflon webpage. [Online]. Available: http://www.emoflon.org/

[8] G. Kulcsár, M. Stein, I. Schweizer, G. Varró, M. Mühlhäuser, and A. Schürr, "Rapid Prototyping of Topology Control Algorithms by Graph Transformation," in GraBaTs, 2014, pp. 1-14. 